\title{
USE OF OPENLY AVAILABLE SATELLITE IMAGES FOR REMOTE SENSING EDUCATION
}

\author{
C.-K. Wang \\ Department of Geomatics, National Cheng Kung University, Tainan, 701, Taiwan \\ chikuei@mail.ncku.edu.tw
}

KEY WORDS: Remote Sensing, Remote Sensing Education, Openly Available Satellite Imagery

\begin{abstract}
:
With the advent of Google Earth, Google Maps, and Microsoft Bing Maps, high resolution satellite imagery are becoming more easily accessible than ever. It have been the case that the college students may already have wealth experiences with the high resolution satellite imagery by using these software and web services prior to any formal remote sensing education. It is obvious that the remote sensing education should be adjusted to the fact that the audience are already the customers of remote sensing products (through the use of the above mentioned services). This paper reports the use of openly available satellite imagery in an introductory-level remote sensing course in the Department of Geomatics of National Cheng Kung University as a term project. From the experience learned from the fall of 2009 and 2010, it shows that this term project has effectively aroused the students' enthusiastic toward Remote Sensing.
\end{abstract}

\section{INTRODUCTION}

With the advent of Google Earth (Google, 2011a), Google Maps (Google, 2011b), and Microsoft Bing Maps (Microsoft, 2011), high resolution satellite imagery are becoming more easily accessible than ever. More importantly, these software or web services are effective and efficient interfaces to provide integrated digital environment for querying, downloading, and displaying openly available satellite imagery on any PC with internet connection. Prior to any formal remote sensing education, the college students may already have wealth experiences using these software and web services, mainly due to their popular functions (such as route planning) and friendly GUI design.

It is obvious that the remote sensing education should be adjusted to the fact that the audience are already the customers of remote sensing products (i.e., route planning while recognizing specific landmark or building in the image). It is easy to introduce remote sensing to them because they are already familiar with the high resolution satellite imagery. And, many of them are expecting to see more pictures of this kind and looking for more applications. However, to the contrary, the curriculum of the remote sensing course usually follows the content of the textbooks, for example, Lillesand et al. (2008) and Campbell (2008), by starting with the introduction of electromagnetic radiation, image interpretation, and active and passive sensors. With the already packed course materials focusing on the Physics aspects of the subject, the application of using high resolution satellite imagery is usually not the core.

To not disappoint the audience and to attract prospective students into the subject of remote sensing, the introductory course, Remote Sensing 1, taught at the Department of Geomatics of National Cheng Kung University has adopted the freely available satellite imagery as a term project assigned to each student, in addition to the course works (mainly laboratory reports). In that project, each student is required to find an area of his/her interest and acquire the satellite imagery, either by sceenshot or downloading the data, for change analysis. Since the concept of image classification will not be covered until the next advanced course, Remote Sensing 2, the change analysis for the term project is only conducted by visual comparison by student's subjective judgement.

\section{SOFTWARE AND DATA}

As the goal of the term project is to encourage students to be exposed to as many satellite images as possible, assigning one particular software would implicitly limit this opportunity. So, the following software is introduced to mitigate the hassles of data pre-processing (colour balancing and georectification). And, the students are highly encouraged to acquire openly available satellite imagery from elsewhere.

\subsection{Google Earth}

The Google Earth is highly acceptable by students due to its intergraded interface, which eliminates the hassle of querying, accessing, downloading, and displaying images. The imagery provided through Google Earth can be recognized in the interface, such as time of acquisition and image source (usually the image distributor). However, it lacks more detailed information, such as sensor name, resolution, whether or not the image has been pan-sharpened, etc.

Although Google Maps is a similar web version of Google Earth, the latter is preferred for the term project because of the Historical Imagery function. Students can easily scroll through time using that function.

The KML and KMZ markup languages, integrated in the Google Earth, also provide a easy means for students to mark the AOI with a placemark or a polygon and for the instructor to load all the AOIs from the students KML files for check-up (details in section 3.1). 

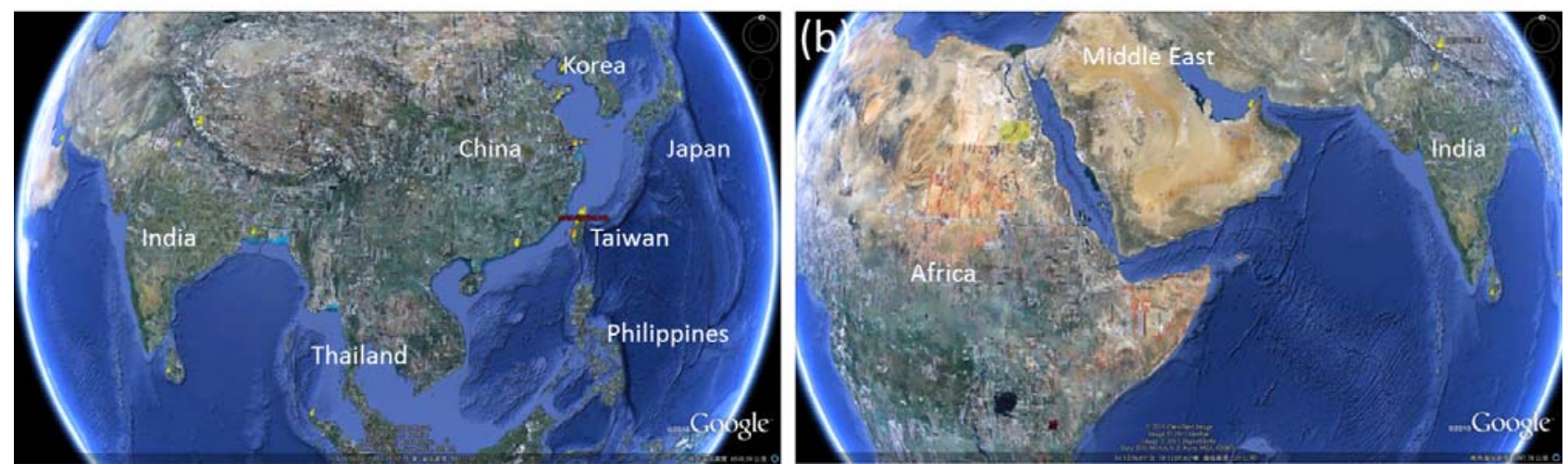

Figure 1. The screenshots showing the AOIs in (a) Asia and (b) Middle East and Africa from the 2009 Fall class. The AOIs are indicated by pins, polygons, or other marks provided in Google Earth.

\subsection{NASA World Wind}

NASA World Wind (Kim, 2006) is similar to Google Earth, but the data provide through it are mainly Landsat and MODIS imagery. It also provides historical images function to access the imagery archive. However, it is found that the bandwidth of NASA World Wind has caused significant delay of image transfer. Thus, this software is rarely used by students.

\subsection{Other software}

Strictly speaking, the development of Google Earth and NASA World Wind are more oriented for land surface applications. For other remote sensing applications, such as oceanography, students can look up the data from MODIS and MERIS. Generating ocean products, such as sea surface temperature or chlorophyll a concentration from these data requires specialized software, such as the SeaDAS (Feldman, 2011) and BEAM (Brockmann Consult, 2011) software provided by NASA and ESA, respectively. For even more remote sensing applications, a comprehensive list of the openly available data released by NASA and the software for processing these data can be found in NASA (2009).

\subsection{Course website}

The course website is created and hosted by the university, integrated into an e-learning platform, with the URL of http://moodle.ncku.edu.tw/. It is stable and capable of handling the submission of the course assignments, where massive data transfer happens when the deadline of the course assignment approaches. The use of the course website is preferred than email submission, where there has been several instances that the submission has been lost or delayed by the email service. The distribution of course materials is also facilitated by the course website.

\section{COURSE ASSIGNMENTS}

During the 18 week semester, there are three course assignments related to the term project.

\subsection{Choose an area of interest and the first report}

The first assignment is to choose an area of interest (AOI) by the student's preference. For a meaningful change analysis, the change on the ground needs to significant enough to be visible in the satellite imagery. An example of Las Vegas, NV, USA, which just experienced a $35 \%$ growth of population, during the last decade (Goldberg, 2011), is demonstrated to the students in the 1st week using Google Earth.

The AOI can be a city, country, continent, forest, or a lake, as long as there is openly available multi-temporal satellite imagery to conduct the change analysis. After deciding his/her AOI, students then submit their AOI as a KML file, which can then be easily brought into Google Earth for check-up.

From the past, it has shown that the choice of AOI is closely related to the focus of mass media. For example, Beijing and Shanghai, China are popular AOIs in the Fall semesters of 2009 and 2010 because of 2008 Olympic and 2010 Expo, respectively. The mega construction projects (The World, and The Palm islands) in Persian Gulf have been the hot choice. Disaster affected areas, such as the southern part of Taiwan that was ruined by Moraka typhoon in 2009 causing more than 600 deaths, is also a frequent site. Figure 1 shows the AOIs from the 2009 Fall class. It is obvious that more AOIs are in Asia, many of which are near Taiwan (Figure 1a), than in Middle East and Africa (Figure 1b).

In addition to the submission of the KML file of AOI, students are required to submit a two A4 page report on the background of the AOI and give some judgments on the satellite imagery they have collected. This is to ensure students have studied the area, knowing what kind of change have occurred during the acquisition of the satellite imagery and knowing what to expect from the change analysis.

It is quite often that many AOIs are focusing on the same area. To prevent too many final presentations with high degree of similarity and to broaden students experience on image interpretation, it is the rule of the course that no more than two AOIs can be the same area. But it is okay to have overlapping AOIs, or have an AOI enclosed by another AOI because the change analysis is likely to be different with different AOI size. So, the check-up of the AOIs is conducted in front of the class. The students, whose AOIs are at the same area, can then arrange their AOIs to other area.

The first assignment is due on the 5th week of the semester.

\subsection{Intermediate report}

After choosing the area of interest, students submit an intermediate report with four A4 pages, which includes the background information of their AOI, the judgment of the 
(a)

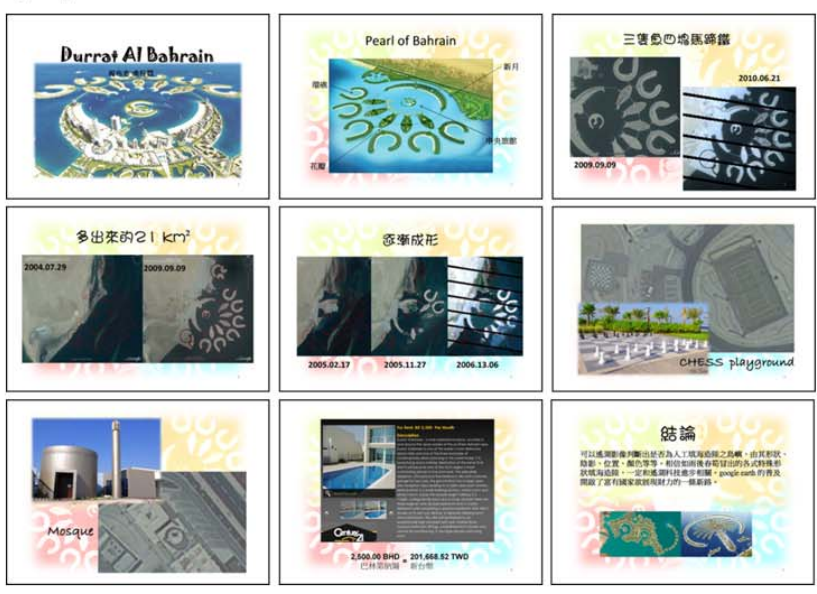

(c)

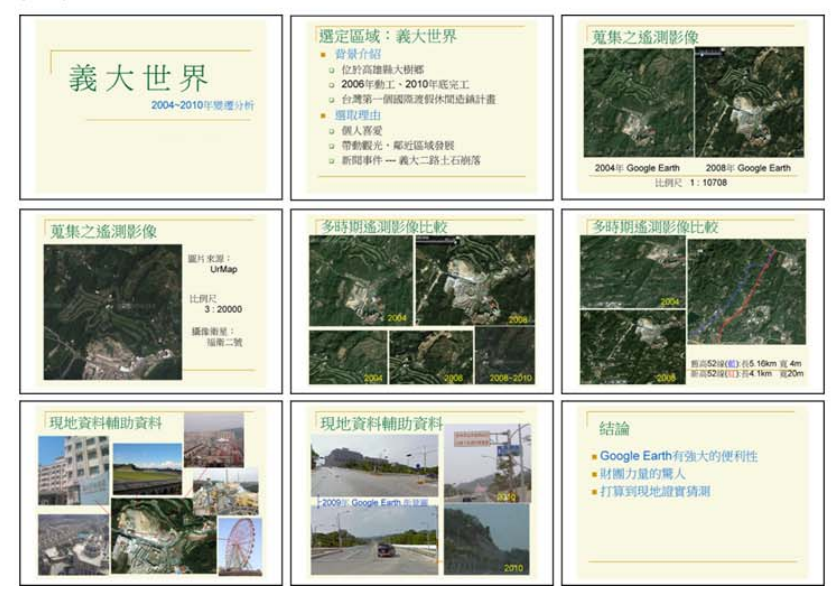

(b)

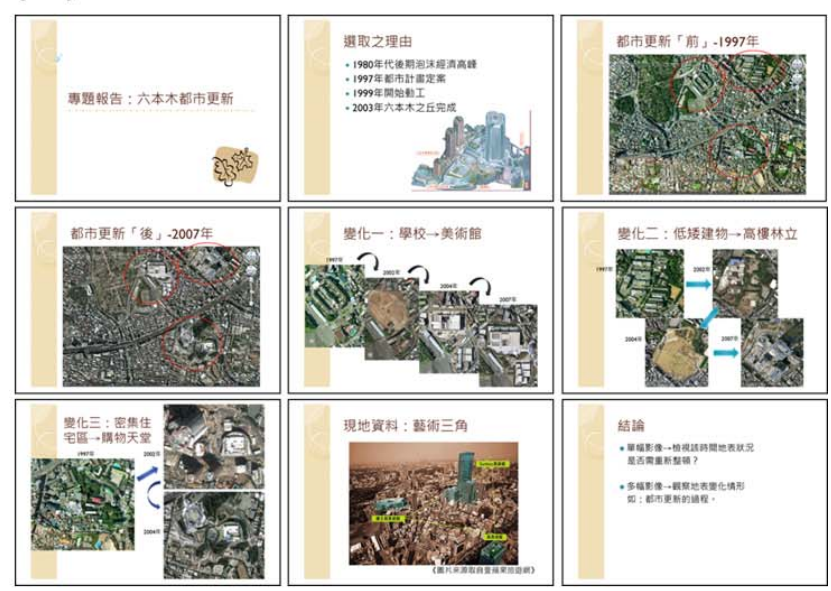

(d)

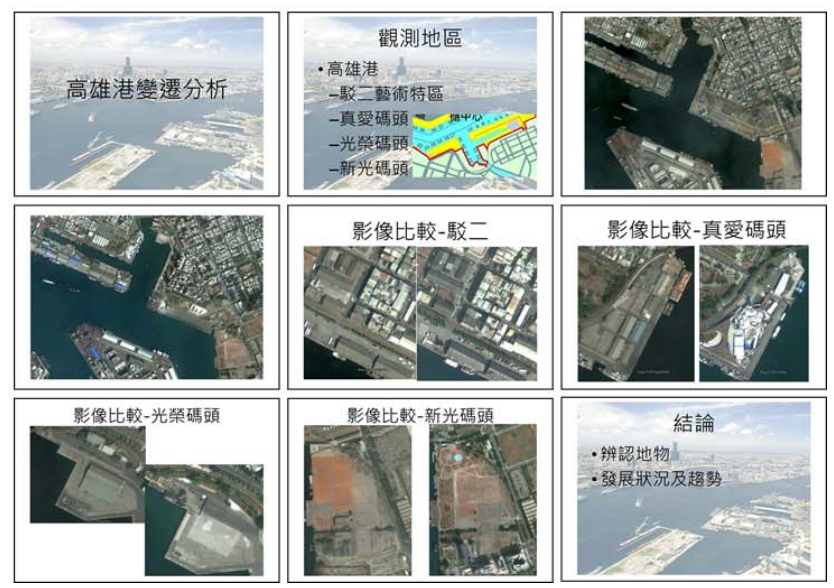

Figure 2 Example final presentations from the 2010 Fall class with the AOI at (a) Durrat Al Bahrain, Kingdom of Bahrain, (b) Roppongi, Tokyo, Japan, (c) A resort at Kaohsiung, Taiwan, and (d) Port of Kaohsiung, Taiwan.

satellite imagery that they have acquired for change analysis, and preliminary change analysis, in the 11th week of the semester. This report is an extended version of that has been submitted in the first assignment. With the introduction of remote sensing theories in the course till then, students can have better judgement on the quality of their satellite imagery than they were at the beginning of the semester. So, at this point, students are given the freedom to change their AOI if they feel necessary. Still, after the submission of the intermediate report, the students can change their AOI, but they are advised not to do so because there isn't much time left ( 5 - 6 weeks) before the final presentation.

\subsection{Final report and presentation}

At the end of the semester (i. e., the 16th and 17th week; the 18th week is designated for final examination), six course hours are allocated for the presentation of the change analysis in front of the class. Normally there are around 50 students enrolled in RS1. Each student gives an 8-minitue presentation with a maximum of 9 slides. All the presentation files are submitted electronically to the course website and are tagged with the time of submission, which was generated by the e-learning system. The order of the final presentation is based on the time tag of the presentation file (first come first serve). Due to the limited time slot available for presentation, there are usually around 10 students who cannot present in front of the class, but have to do it afterward to the instructor.

The contents of all the presentations, especially those conducted in front of the class, are included in the final examination, which is an open book test. The 9 slides of each presentation can be easily fitted in one A4 page with a 3 by 3 arrangement. All of presentation slide are then combined into a single .pdf file and made available to the students. Figure 2 shows four final presentations from the 2010 Fall class. In Figure 2a, the student shows the emergence of a man-made island at Durrat $\mathrm{Al}$ Bahrain, Kingdom of Bahrain. In Figure 2b, the student shows the renovation process of Roppongi, Tokyo, Japan. In Figure 2c, the student shows the construction progress of a resort at Kaohsiung, Taiwan. In Figure 2d, the student shows the regalvanization of the disserted land of the Port of Kaohsiung, Taiwan.

There is no need to memorise the details of the presentation, but students do need to evaluate the strengths and weaknesses and possible mistakes made by their classmates. Students can bring a copy of all the presentations to the examination. The normal questions in the final examination regarding the final presentation are as follows. 
Considering the final presentations that were able to be presented in class, please divide these presentations into three groups (supreme, medium, and below average) according to their quality (completeness of the presentation, logical thought, wealth of image collection, image interpretation, and other criteria by your judgment).

1. Please give a list of 10 names of the presenter who gave a supreme presentation.

2. Please give a list of 10 names of the presenter who gave a medium presentation.

3. Please give a list of 5 names of the presenter whose presentation is below average.

4. Pick any one from the supreme group, why do think his/her presentation is of supreme quality?

5. Pick any three from the below average group, give two suggestions for each presentation to improve his/her presentation (i.e., there should be a total of 6 suggestions).

This set of questions is quite effective to tell the degree of participation of students of this project. For example, from question 1 to 3 , there requires a list of 25 names. If the student is physically or mentally absent during the presentation, he/she can be easily identified when a name (usually more than one) is misplaced in the three groups.

A final report, no more than 6 A4 pages, covering the complete change analysis of the AOI is required in addition to the presentation in the 16th week.

\section{CONCLUSIONS}

This paper reports the use of openly available satellite imagery in an introductory-level remote sensing course in the Department of Geomatics of National Cheng Kung University as a term project. From the experience learned from the fall of 2009 and 2010, it shows that this term project has effectively aroused the students' enthusiastic toward Remote Sensing.

\section{REFERENCES}

National Aeronautics and Space Administration, 2009. "Earth System Science Data Resources ", http://earthdata.nasa.gov/sites/default/files/field/document/ESS DR2009.pdf (accessed 29 August, 2011)

Campbell, J. B., 2008. Introduction to remote sensing. 4th ed. New York: Guildford Press, pp. 626

Brockmann Consult, 2011. "Welcome to BEAM", http://www.brockmann-consult.de/cms/web/beam/ (29 August 2011)

Feldman, G. C., 2011. "SeaDAS Home Page", http://seadas.gsfc.nasa.gov/ (accessed 29 August, 2011)

Goldberg, D., 2011. "Census: Las Vegas Valley boom fueled Nevada’s record growth." In Las Vegas Sun. Las Vegas, NV

Google, 2011a. "Google Earth", http://www.google.com/earth/index.html (accessed 29 August, 2011)
Google, 2011b. "Google Maps", http://maps.google.com/ (accessed 29 August, 2011)

Kim, R., 2006. "NASA World Wind", http://worldwind.arc.nasa.gov/index.html (accessed 29 August, 2011)

Lillesand, T. M., R. W. Kiefer, and J. W. Chipman, 2008. Remote sensing and image interpretation. 6th ed. Hoboken, NJ: John Wiley \& Sons, pp. 756

Microsoft, 2011. "Bing Maps", http://www.bing.com/maps/ (accessed 29 August, 2011) 
International Archives of the Photogrammetry, Remote Sensing and Spatial Information Sciences, Volume XXXVIII-6/W27, 2011 ISPRS Taipei 2011 Workshop, 4-6 October 2011, Taipei, Taiwan 
International Archives of the Photogrammetry, Remote Sensing and Spatial Information Sciences, Volume XXXVIII-6/W27, 2011 ISPRS Taipei 2011 Workshop, 4-6 October 2011, Taipei, Taiwan

Administration, National Aeronautics and Space. 2009. "Earth System Science Data Resources ". http://earthdata.nasa.gov/sites/default/files/field/docu ment/ESSDR2009.pdf.

Campbell, James B. 2008. Introduction to remote sensing. 4th ed. New York: Guildford Press.

Lillesand, Thomas M., Ralph W. Kiefer, and Jonathan W. Chipman. 2008. Remote sensing and image interpretation. 6th ed. Hoboken, NJ: John Wiley \& Sons. 